\title{
Innovative Maschinen- und Antriebskonzepte
}

im Lichte der Kooperation zwischen Universität
und Industrie unter Berücksichtigung der neuen
Universitätsstruktur entsprechend dem UOG 2002

Es ist eine bekannte Tatsache, dass etwa die Hälfte der „erzeugten" elektrischen Energie mit Hilfe elektrischer Maschinen und Antriebe wieder in mechanische Energie umgewandelt wird.

Ebenso bekannt - jedoch nicht immer beachtet und gebührend bewundert - sind die enormen Bereiche von etwa neun Zehnerpotenzen hinsichtlich der umgesetzten Leistungen ( $\mathrm{mW}$ bis MW) sowie der sechs bis sieben Zehnerpotenzen hinsichtlich der vorkommenden Drehzahlen (wenige Umdrehungen pro Minute bis hunderttausend Umdrehungen pro Minute und mehr).

Die technische Entwicklung der elektrischen Antriebe verlief naturgemäß von den stationären Antrieben unter Einsatz aller verfügbaren elektrischen Maschinen zu den zunächst steuerbaren, über die regelbaren Antriebe bis hin zu den höchst anspruchsvollen Antriebssystemen in den verschiedensten Formen der Industrie.

Möglich geworden ist diese Entwicklung durch die Fortschritte in den „umliegenden" Fachgebieten: Berechnung, Konstruktion und Bau elektrischer Maschinen, Erforschung und Realisierung von Elementen der Leistungselektronik und deren Anwendung in der Stromrichtertechnik, Verbesserung der Steuerungselektronik und Erhöhung der verfügbaren Rechenleistung von Mikro- und Signalprozessoren sowie der kontinuierlichen verbesserten Anpassung durch Modellbildung und Simulation, also der regelungstechnischen Komponenten. Daraus ist unschwer zu erkennen, dass die elektrische Antriebstechnik immer mehr zu einem fachübergreifenden Wissensgebiet geworden ist. Das ständige Bemühen nach einer optimalen Anpassung der elektrischen Maschine an die Erfordernisse der Arbeitsmaschine bzw. des Arbeits- und Herstellungsprozesses durch Zuhilfenahme der Leistungselektronik und Regelungstechnik macht es notwendig, dass bei der Konzeption eines elektrischen Antriebs auch die konkreten Erfahrungen des Betriebs, das heißt also die Praxis mitherangezogen werden müssen.

Aus diesen Gründen werden seit dem Jahr 2001 Tagungen zum Thema: „Elektrische Maschinen und Antriebe" gemeinsam von den Technischen Universitäten Wien und Graz mit dem Ziel durchgeführt, nicht nur die institutsübergreifende Forschung und Entwicklung zu intensivieren, sondern auch deren Ergebnisse direkt an die Betreiber weiterzugeben und umgekehrt ebenso direkt die Erkenntnisse und Erfahrungen der Industrie umgehend wieder zurückzuführen.

Derartige Kooperationen zwischen Universität und Industrie sind überaus sinnvoll und wurden im Rahmen der Teilrechtsfähigkeit nach dem bis Ende 2003 gültigen UOG 93 auch erfolgreich durchgeführt.

Mit dem Wirksamwerden des UOG 2002 am 1. Jänner dieses Jahres sind die Universitäten Österreichs in die so genannte Vollrechtsfähigkeit übergeleitet worden, wobei der Begriff „Autonomie“ als augenscheinlicher Ausdruck für den lang ersehnten Wunsch der Universitäten, vom Gängelband des Ministeriums loszukommen, verwendet wurde und wird.

Dass hinter dieser Vorgangsweise große Gefahren stecken, ist inzwischen vielen Betroffenen in und Nichtbetroffenen außerhalb der Universität deutlich geworden.

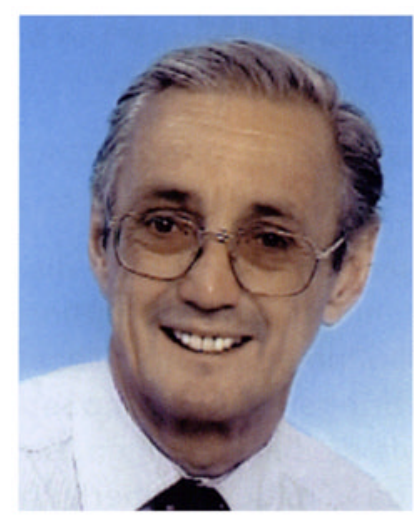

Die finanzielle Grundversorgung - auch nach UOG 2002 eine Verpflichtung des Staates - ist bereits im Jahr 2003 drastisch zurückgegangen und wird auch bei optimistischer Einschätzung in den nächsten Jahren nicht wieder wesentlich ansteigen.

Technisch-naturwissenschaftliche Fakultäten sind daher in immer stärkerem Maße gezwungen, Mittel aus Projekten in die Infrastruktur der Institute zu stecken, das heißt Anschaffungen zu tätigen, die früher in die Budgetzuweisung des Bundes gefallen sind.

Extrem schwierig und auf längere Sicht durchaus gefährlich ist in diesem Zusammenhang die Entlohnung junger Assistenten, welche so gering ist, dass praktisch kaum Anreiz besteht, die berufliche Laufbahn an der Universität zu beginnen.

Um überhaupt wissenschaftlichen Nachwuchs zu bekommen, wird daher ebenfalls auf Projekteinnahmen zurückgegriffen - obwohl diese Vorgangsweise nur unter Umgehung der gesetzlichen Bestimmungen möglich ist.

Was machen aber Institute mit geringen Drittmitteln, wie es naturgemäß bei grundlagenorientierten und theoretischen Fachgebieten der Fall ist? Diese laufen Gefahr, wegen nicht nachbesetzbarer Stellen wissenschaftlicher Mitarbeiter auszutrocknen, obwohl gerade sie unverzichtbare Basis jeder universitärer Forschung und Entwicklung sind.

Immer öfter und mit stärkerer Akzentuierung kann man daher in Kommentaren von der Bedrohung der Grundlagenforschung durch das Entstehen „unternehmerischer" Universitäten lesen.

Auf der anderen Seite sind diese „unternehmerischen“ Universitäten gezwungen, auf stärkere Betonung anwendungsorientierter und somit wirtschaftsnäherer Forschung überzugehen und laufen Gefahr, die gegenüber dem Ministerium gewonnene Autonomie an die Wirtschaft mit deren weitgehend rein materiellen Zielen wieder zu verlieren.

Nach einer beinahe zehnjährigen Amtszeit als Dekan der Fakultät für Elektrotechnik und Informationstechnik der Technischen Universität Graz erlaube ich mir, auf diese Tatbestände mit der Bitte hinzuweisen, dass diese von den meist neuen Funktionären der „vollrechtsfähigen“ Universitäten auch entsprechend erkannt und deren negativen Auswirkungen hintangehalten werden mögen.

\section{O. Univ.-Prof. Dipl.-Ing. Dr. techn. Manfred Rentmeister}

Vorstand des Instituts für Elektrische Antriebe und Maschinen der Technischen Universität Graz 